\title{
Discrimination of a selected set of turmeric, ginger, fenugreek and coriander varieties using ISSR markers
}

\author{
A. Giridhari, I.P. Vijesh Kumar and T.E. Sheeja* \\ ICAR-Indian Institute of Spices Research, Kozhikode-673 012, Kerala, India
}

(Manuscript Received: 28-02-2020, Revised: 20-07-2020, Accepted: 08-09-2020)

\begin{abstract}
DNA fingerprints are unique to individuals and can be used to identify individuals as in the case of conventional fingerprints. Plant DNA fingerprinting make use of various molecular markers for identifying newly released crop varieties and are all the more important in plant variety registration under the PPV\&FR Act of 2001. The trade-related intellectual property rights (TRIPS) and the convention on biological diversity (CBD) insist on the establishment of identity and ownership of genotypes for enforcement of their provisions for securing protection to plant varieties as well as for regulating access to germplasm resources. DNA fingerprints, along with morphological markers, can be efficiently utilized for plant varietal identification, detection of duplicates and adulterants. Here in this particular study, the spice samples received at the DNA fingerprinting facility (DNAFF) of ICARIndian Institute of Spices Research (ICAR-IISR) from various centres of All India Coordinated Project on Spices (AICRPS) were DNA fingerprinted using inter simple sequence repeat (ISSR) markers. The DNA profile of a candidate variety vis-a-vis check variety is an essential prerequisite during submission of proposal for release of crop variety to central sub-committee on crop standards notification and release of varieties. The new varieties of turmeric, ginger, coriander and fenugreek were compared with the closely resembling check varieties for establishing distinctness for varietal registration. A total of 118 ISSR primers were screened in the above-given crops, to identify the distinct markers identifying the candidate from the check varieties. Using this technique, the DNAFF at ICAR-IISR could facilitate registration of turmeric varieties, Roma, Rasmi and Suroma; ginger varieties Suruchi, Suravi and Suprabha; coriander varieties, Suguna, Susthira and Suruchi, while varieties of turmeric, Uttara Rupanjana and Uttara Ranjini; fenugreek variety Ajmer fenugreek (AFg-5); coriander varieties Ajmer coriander (ACr-2) and Chhattisgarh Shri Chandra Hasini dhaniya-2 (ICS-4) are in the process of getting registration. ISSR markers were found to be appropriate for establishing distinctness of the new varieties of spices for securing varietal registration.
\end{abstract}

Keywords: Coriander, DNA fingerprinting, fenugreek, ginger, ISSR, turmeric

\section{Introduction}

Sir Alec Jeffreys developed the concept of DNA fingerprinting in 1985 (Jeffreys et al., 1985) for the detection of highly variable DNA fragments by hybridisation using multilocus probes. These DNA fingerprints, resembling barcodes are unique to individuals and can be used as the conventional fingerprints to identify individuals with absolute certainty. The genetic profile of a sample is compared with the known set of a library of reference fingerprints to find out the one which is closest to the sample.

Plant DNA fingerprinting make use of molecular markers to identify cultivars. Conventional systems like morphological or biochemical markers have got various drawbacks like the influence of environmental factors, epistatic interactions, pleiotropic effects etc., which can affect the efficiency of these systems in unambiguously identifying the varieties from one another. Similarly, there exists a need for a large number of morphological descriptors that allow the identification of the increasing number of varieties. DNA based markers are also more stable and not affected by external environmental conditions. It has got the advantage that these markers can be made use of at any developmental stage of the plant, unlike the conventional systems that are applicable only

*Corresponding Author: sheeja@spices.res.in 
at a specific stage of the plant. Since these markers make use of the basic genomic information of the plants, they are more reliable and reproducible. The advent of next-generation sequencing (NGS) technologies has drastically cut down the cost of analysis, making fingerprinting more accessible to a wide range of crops.

Due to rapid progress in varietal development, it has become increasingly difficult to differentiate varieties based on their phenotypic characters alone, especially the improved varieties. DNA fingerprinting is hence used by plant breeders of both private and public sectors for identification of crop varieties and many research organizations in India and across the globe have started offering DNA testing for plant varietal identification. More importantly, Protection of Plant Variety and Farmers Right Authority (PPV \& FRA, 2001), Govt. of India, has made DNA fingerprinting as a mandatory requirement for new crop varieties released by the central varietal release committee (Shivakumar et al., 2014). DNA fingerprinting as a tool can be used not only by geneticists but also by economists and social scientists for gaining information about varietal use by farmers and varietal turnover and rates of diffusion of new varieties. DNA fingerprinting has emerged as a robust tool in the identification of newly developed crop varieties and plays a key role in protecting plant breeder's rights as per the PPV \& FR Act. It also helps in testing the authenticity of crops.

A review by Nybom et al. (2014) based on 292 papers published between mid-2006 and mid-2009 on marker-based studies for plant varietal discrimination, showed that the locus-specific microsatellite analysis (SSR) is the most popular method (36\%), followed by RAPD (27\%), ISSR (13\%), AFLP (11\%), other nuclear DNA-based methods $(10 \%)$, like CAPS, DAMD, IRAP, REMAP, SNPs, SCAR, SRAP and organellar DNA-based methods (3\%), which mostly includes chloroplast (cpDNA) based methods. NRC on DNA fingerprinting, NBPGR, New Delhi has fingerprinted over two thousand varieties, parental lines and hybrids of 32 important crops using STMS, AFLP, ISSR and RAPD techniques (Bhat, 2006). But the major constraint in the area is the development of standardised protocols for carrying out fingerprinting analysis as well as finding out suitable, robust and reliable marker systems for each crop that can be effectively used across labs.

Inter SSR (ISSR) fingerprinting was developed as no prior sequence knowledge is required. ISSR technique provides a quick, reliable and highly informative system for DNA fingerprinting. ISSR markers are inherited in Mendelian mode and segregated as dominant markers. Primers based on a repeat sequence, such as (CA)n can be made with a degenerate 3'-anchor, such as $(\mathrm{CA})_{8} \mathrm{RG}$ or $(\mathrm{AGC})_{6}$ TY. The resultant PCR reaction amplifies the sequence between two SSRs, yielding a multilocus marker system useful for fingerprinting, diversity analysis and genome mapping (Godwin et al., 1997). ISSR markers have been mostly used either alone or in combination with other markers like SSR, RAPD, AFLP etc., for genetic diversity and relationship and cultivar identification studies (Abdulla and Gamal 2010; Costa et al., 2016; Torre et al., 2012; Wu et al., 2009). This technique has been widely used in the studies of cultivar identification, genetic mapping, gene tagging, genetic diversity, evolution and molecular ecology (Wang, 2002). DNA fingerprinting in Acacia using ISSR primers showed that a total of 71 bands of 70 bp to 2,200 bp were amplified, with an average polymorphism information content per primer of 77 . The primers were successful in distinguishing Acacia spp (Alhasnawi et al., 2019). Cultivars of sweet potato were identified using ISSR (McGregor et al., 2000). In durum wheat, two primers were found sufficient to distinguish 52 durum wheat cultivars and breeding lines indicating the very good discriminating ability of ISSR techniques (Pasqualone et al., 2000). In date palm, accurate fingerprints were generated to distinguish cultivars from each other (Sabir et al., 2014). Species-specific bands were identified in mulberry using ISSR (Awasthi et al., 2004). DNA fingerprinting of released varieties and selected superior somaclones (two released varieties and four selected superior somaclones) of ginger (Zingiber officinale Rosc.) were carried out using eleven ISSR primers. The primers were able to distinguish the released variety Athira from the parent variety, and the somaclone 
$292 \mathrm{R}$ was found to be diverse than the source parent Rio-de-Janeiro (Gosh, 2013).

ICAR-Indian Institute of Spices Research (ICAR-IISR), houses the biggest repository of spices in the world. More than 5000 accessions are maintained in the germplasm repository of ICARIISR, including black pepper (3181), ginger (668) and turmeric (1404). ICAR-IISR has so far released 25 high yielding spice varieties including that of black pepper (8), ginger (3), turmeric (7), cardamom (3), cinnamon (2) and nutmeg (2) (http:// www.spices.res.in/research-highlights). Being a premier organisation dedicated to spices and due to the reason that DNA fingerprints are mandatory for varietal registration, there is a constant request from the various All India Coordinated Research Project on Spices (AICRPS) centres for facilitating fingerprinting of their new varieties of spices. A recently established DNA fingerprinting facility at ICAR-IISR is now serving as a nodal centre for fingerprinting of major spices like black pepper, turmeric, ginger, cardamom, nutmeg and seed spices like fennel, fenugreek, coriander, celery and cumin from the AICRPS centres all over the country. At the facility, we are also trying to evolve suitable molecular marker systems to identify parental lines, landraces and wild relatives along with released varieties to enforce the propriety rights over varieties and germplasm of major spices. A combination of morphological and DNA-based markers is efficiently used as a reliable method for the identification of varieties and testing their authenticity. In this particular work ISSR markers were used to distinguish between different varieties of crops like turmeric, ginger, fenugreek and coriander from various AICRPS centres for facilitating varietal release through Central Varietal Release Committee (CVRC). ISSR markers are used because no sequence information is required, and they show a higher level of polymorphism than RAPD or RFLP markers (Godwin et al., 1997).

\section{Materials and methods}

The candidate varieties of spice crops like turmeric, ginger, coriander and fenugreek for DNA profiling was received from various centres of the AICRPS. The total genomic DNA was isolated from the plant samples, either from the leaf tissue or from the seeds. DNA isolation was carried out either using the genomic DNA isolation kit (Sigma-Aldrich) in case of leaf tissues or manually using CTAB method from the seed samples of with slight modifications (Doyle and Doyle, 1987; Swetha et al., 2014). The quantity and quality of DNA were analysed using 0.8 per cent agarose gel and bio-photometer readings (Eppendorf). ISSR profiling was done using PCR with template DNA (10-50 ng) and Emerald green PCR master mix (Takara). The PCR products were analysed on 2 per cent agarose gels. The gels were run at $80 \mathrm{~V}$ for $2-2.5 \mathrm{~h}$ and visualized using gel documentation unit. The ISSR profiles of candidate variety were compared with its closely resembling check variety, and the distinct marker bands were identified. A total of 118 primers were screened in all the spice varieties with more than 25 primers screened in each case to identify the distinct marker. The primer names and sequence details are provided in Table 1 and details of the source of spice samples are provided in Table 2 .

\section{Results and discussion}

Total genomic DNA was isolated from either leaf tissue or seeds using a genomic DNA isolation kit or modified CTAB method. The seeds have a greater level of protein and polysaccharide contaminants that hinder the downstream PCR reactions in case of coriander. These contaminants were overcome by increasing the concentration of CTAB (4\%) and sodium chloride to $3 \mathrm{M}$ in the extraction buffer and by the addition of sodium acetate during chloroform extraction procedure (Swetha et al., 2014). The quality of extracted DNA was analysed in 0.8 per cent agarose gel. The DNA was obtained without any shearing. The biophotometer readings were also analysed, and the A260/280 values were found to be in the range of 1.8-2.0, which indicates that the DNA was free from RNA and protein contamination. This good quality DNA was further diluted and used for PCR using ISSR primers.

The DNA profiles of candidate variety and the check variety were compared, and the distinct marker was identified. Among the 118 ISSR primers screened in total, only six were found to be useful in distinguishing the candidate variety from the check. 
Table 1. List of primers used for ISSR profiling in spices

\begin{tabular}{|c|c|c|c|}
\hline $\begin{array}{l}\text { Sl. } \\
\text { No. } \\
\end{array}$ & Primer name & Sequence (5'-3' motif) & Crops profiled \\
\hline 1. & $(\mathrm{AAG})_{5} \mathrm{CC}$ & AAGAAGAAGAAGAAGCC & Coriander \\
\hline 2. & $(\mathrm{AAG})_{5} \mathrm{GC}$ & AAGAAGAAGAAGAAGGC & Coriander \\
\hline 3. & $(\mathrm{AGG})_{6}$ & AGGAGGAGGAGGAGGAGG & Turmeric, Ginger, Fenugreek \\
\hline 4. & $(\mathrm{AGTG})_{7} \mathrm{G}$ & AGTGAGTGAGTGAGTGAGTGAGTGAGTGG & Fenugreek \\
\hline 5. & $(\mathrm{AT})_{7} \mathrm{G}$ & ATATATATATATATG & No amplification \\
\hline 6. & $(\mathrm{CA})_{7} \mathrm{AC}$ & CACACACACACACAAC & Coriander \\
\hline 7. & $(\mathrm{CA})_{7} \mathrm{AG}$ & CACACACACACACAAG & Coriander \\
\hline 8. & $(\mathrm{CA})_{7} \mathrm{GG}$ & CACACACACACACAGG & Coriander \\
\hline 9. & $(\mathrm{CA})_{7} \mathrm{GT}$ & CACACACACACACAGT & Coriander \\
\hline 10. & $(\mathrm{CAA})_{5}$ & CAACAACAACAACAA & Turmeric, Ginger, Fenugreek, Coriander \\
\hline 11. & $(\mathrm{CT})_{8} \mathrm{AC}$ & СТСТСТСТСТСТСТСТАС & Coriander \\
\hline 12. & $(\mathrm{CT})_{8} \mathrm{CC}$ & СТСТСТСТСТСТСТСТСС & Turmeric, Ginger, Fenugreek \\
\hline 13. & $(\mathrm{CT})_{8} \mathrm{GC}$ & стСТСТСТСТСТСТСТGC & Coriander \\
\hline 14. & $(\mathrm{CTC})_{8} \mathrm{GC}$ & СТССТССТССТССТССТССТССТСGC & Coriander \\
\hline 15. & $(\mathrm{GA})_{6} \mathrm{CC}$ & GAGAGAGAGAGACC & Coriander \\
\hline 16. & $(\mathrm{GA})_{6} \mathrm{GG}$ & GAGAGAGAGAGAGG & Coriander \\
\hline 17. & $(\mathrm{GA})_{9} \mathrm{~T}$ & GAGAGAGAGAGAGAGAGAT & Ginger, Coriander \\
\hline 18. & $(\mathrm{GACA})_{3} \mathrm{GG}$ & GACAGACAGACAGG & No amplification \\
\hline 19. & $(\mathrm{GACA})_{4} / \mathrm{ISSR} 03$ & GACAGACAGACAGACA & Turmeric, Ginger \\
\hline 20. & $(\mathrm{GATA})_{3} \mathrm{CC}$ & GATAGATAGATACC & No amplification \\
\hline 21. & $(\mathrm{GGA})_{4}$ & GGAGGAGGAGGA & Turmeric, Ginger, Coriander \\
\hline 22. & $(\mathrm{GGC})_{5} \mathrm{AT}$ & GGCGGCGGCGGCGGCAT & Fenugreek \\
\hline 23. & $(\mathrm{GT})_{6} \mathrm{GG}$ & GTGTGTGTGTGTGG & Coriander \\
\hline 24. & $(\mathrm{GTG})_{5}$ & GTGGTGGTGGTGGTG & Turmeric, Ginger, Fenugreek \\
\hline 25. & (TA)7A & TATATATATATATAA & No amplification \\
\hline 26. & IS $1-(\mathrm{CAC})_{7} \mathrm{~T}$ & CACCACCACCACCACCACCACT & Turmeric \\
\hline 27. & IS 10- BDBT(CCT) & ВDВТССТССТССТССТССТССТ & Turmeric \\
\hline 28. & IS 11- HVH(TCC) 6 & НVHТССТССТССТССТССТСС & Turmeric \\
\hline 29. & IS 2- $(\mathrm{GA})_{9} \mathrm{C}$ & GAGAGAGAGAGAGAGAGAC & Coriander \\
\hline 30. & IS 4- $(\mathrm{CAC})_{7} \mathrm{G}$ & CACCACCACCACCACCACCACG & No amplification \\
\hline 31. & ISSR $01-(\mathrm{CA})_{7} \mathrm{~A}$ & CACACACACACACAA & Turmeric, Ginger, \\
\hline 32. & ISSR 02- (AGTG) ${ }_{3}$ & AGTGAGTGAGTG & Turmeric, Ginger, Fenugreek, Coriander \\
\hline 33. & ISSR $05-(\mathrm{CT})_{7} \mathrm{TG}$ & СТСТСТСТСТСТСТТG & Turmeric, Ginger, Fenugreek, Coriander \\
\hline 34. & ISSR 07- $(\mathrm{GA})_{8} \mathrm{G}$ & GAGAGAGAGAGAGAGAG & Turmeric, Ginger, Fenugreek, Coriander \\
\hline 35. & ISSR 11- (GACA) & GACAGACAGACA & Turmeric, Ginger, Fenugreek, Coriander \\
\hline 36. & ISSR 12- $(\mathrm{CAC})_{3} \mathrm{GC}$ & CACCACCACGC & Turmeric, Ginger, Fenugreek, Coriander \\
\hline 37. & ISSR 13- (AGTG) ${ }_{3}$ GG & AGTGAGTGAGTGGG & Turmeric, Ginger, Fenugreek, Coriander \\
\hline 38. & ISSR 14- (AGC) $)_{4} \mathrm{GT}$ & AGCAGCAGCAGCGT & Turmeric, Ginger \\
\hline 39. & ISSR 15- $(\mathrm{TCC})_{5}$ & ТССТССТССТССТСС & Turmeric, Ginger \\
\hline 40. & UBC $801-(\mathrm{AT})_{8} \mathrm{~T}$ & ATATATATATATATATT & No amplification \\
\hline
\end{tabular}




\begin{tabular}{|c|c|c|c|}
\hline 41. & UBC 802- $(\mathrm{AT})_{8} \mathrm{G}$ & ATATATATATATATATG & No amplification \\
\hline 42. & UBC $803-(\mathrm{AT})_{8} \mathrm{C}$ & ATATATATATATATATC & No amplification \\
\hline 43. & UBC 804- (TA) $)_{8} \mathrm{~A}$ & TATATATATATATATAA & No amplification \\
\hline 44. & $\mathrm{UBC} 805-(\mathrm{TA})_{8} \mathrm{C}$ & TATATATATATATATAC & No amplification \\
\hline 45. & UBC $806-(\mathrm{TA})_{8} \mathrm{G}$ & TATATATATATATATAG & No amplification \\
\hline 46. & $\mathrm{UBC} 807-(\mathrm{AG})_{8} \mathrm{~T}$ & AGAGAGAGAGAGAGAGT & Turmeric, Ginger, Coriander \\
\hline 47. & UBC 808- $(\mathrm{AG})_{8} \mathrm{C}$ & AGAGAGAGAGAGAGAGC & Turmeric, Coriander \\
\hline 48. & UBC 809- $(A G)_{8} G$ & AGAGAGAGAGAGAGAGG & Turmeric, Ginger, Fenugreek, Coriander \\
\hline \multirow[t]{2}{*}{49.} & UBC $810-(\mathrm{GA})_{8} \mathrm{~T} /$ & & \\
\hline & ISSR 08/ISSR 16/IS 14 & GAGAGAGAGAGAGAGAT & Turmeric, Ginger, fenugreek, Coriander \\
\hline \multirow[t]{2}{*}{50.} & $\mathrm{UBC} 811-(\mathrm{GA})_{8} \mathrm{C} /$ & & \\
\hline & ISSR 06 & GAGAGAGAGAGAGAGAC & Turmeric, Ginger, Fenugreek, Coriander \\
\hline 51. & UBC 812- $(\mathrm{GA})_{8} \mathrm{~A}$ & GAGAGAGAGAGAGAGAA & Turmeric, Ginger, Fenugreek, Coriander \\
\hline 52. & UBC 813- $(\mathrm{CT})_{8} \mathrm{~T} / \mathrm{IS} 19$ & СТСТСТСТСТСТСТСТТ & Coriander \\
\hline 53. & $\mathrm{UBC} 814-(\mathrm{CT})_{8} \mathrm{~A}$ & СТСТСТСТСТСТСТСТА & Coriander \\
\hline \multirow[t]{2}{*}{54.} & UBC $815-(\mathrm{CT})_{8} \mathrm{G} /$ & & \\
\hline & ISSR 09 & СТСТСТСТСТСТСТСТG & Turmeric, Ginger, Coriander \\
\hline 55. & UBC $816-(C A)_{8} T$ & CACACACACACACACAT & Ginger, Coriander \\
\hline 56. & UBC 817- $(\mathrm{CA})_{8} \mathrm{~A} / \mathrm{IS} 20$ & CACACACACACACACAA & Ginger, Fenugreek, Coriander \\
\hline \multirow[t]{2}{*}{57.} & $\mathrm{UBC} 818-(\mathrm{CA})_{8} \mathrm{G} /$ & & \\
\hline & ISSR 17 & CACACACACACACACAG & Turmeric, Ginger, Fenugreek, Coriander \\
\hline 58. & UBC 819- $(\mathrm{GT})_{8} \mathrm{~A}$ & GTGTGTGTGTGTGTGTA & Fenugreek \\
\hline 59. & $\mathrm{UBC} 820-(\mathrm{GT})_{8} \mathrm{C}$ & GTGTGTGTGTGTGTGTC & Coriander \\
\hline 60. & $\mathrm{UBC} 821-(\mathrm{GT})_{8} \mathrm{~T}$ & GTGTGTGTGTGTGTGTT & Coriander \\
\hline 61. & UBC 822- (TC) ${ }_{8} \mathrm{~A} / \mathrm{IS} 25$ & ТСТСТСТСТСТСТСТСА & Fenugreek, Coriander \\
\hline 62. & UBC $823-(\mathrm{TC})_{8} \mathrm{C}$ & ТСТСТСТСТСТСТСТСС & Fenugreek, Coriander \\
\hline 63. & UBC 824- (TC) $)_{8} \mathrm{G}$ & ТСТСТСТСТСТСТСТСG & Fenugreek, Coriander \\
\hline 64. & UBC $825-(\mathrm{AC})_{8} \mathrm{~T}$ & ACACACACACACACACT & Coriander \\
\hline \multirow[t]{2}{*}{65.} & $\mathrm{UBC} 826-(\mathrm{AC})_{8} \mathrm{C} /$ & & \\
\hline & ISSR 04/IS-29 & ACACACACACACACACC & Turmeric, Ginger, Fenugreek, Coriander \\
\hline \multirow[t]{2}{*}{66.} & $\mathrm{UBC} 827(\mathrm{AC})_{8} \mathrm{G} /$ & & \\
\hline & ISSR 10 & ACACACACACACACACG & Turmeric, Ginger, Coriander \\
\hline 67. & UBC 828- (TG) $)_{8} \mathrm{~A}$ & TGTGTGTGTGTGTGTGA & Coriander \\
\hline 68. & UBC 829- $(\mathrm{TG})_{8} \mathrm{C}$ & TGTGTGTGTGTGTGTGC & Coriander \\
\hline 69. & UBC $830-(\mathrm{TG})_{8} \mathrm{G}$ & TGTGTGTGTGTGTGTGG & Coriander \\
\hline 70. & UBC 831- (AT) $)_{8} \mathrm{YA}$ & ATATATATATATATATYA & No amplification \\
\hline 71. & $\mathrm{UBC} 832-(\mathrm{AT})_{8} \mathrm{YC}$ & ATATATATATATATATYC & No amplification \\
\hline 72. & UBC 833- $(\mathrm{AT})_{8} \mathrm{YG}$ & ATATATATATATATATYG & No amplification \\
\hline \multirow[t]{2}{*}{73.} & UBC 834- $(\mathrm{AG})_{8} \mathrm{YT} /$ & & \\
\hline & IS-33 & AGAGAGAGAGAGAGAGYT & Ginger, Coriander \\
\hline 74. & $\mathrm{UBC} 835-(\mathrm{AG})_{8} \mathrm{YC}$ & AGAGAGAGAGAGAGAGYC & Turmeric, Ginger, Coriander \\
\hline 75. & UBC 836- $(\mathrm{AG})_{8} \mathrm{YA}$ & AGAGAGAGAGAGAGAGYA & Turmeric, Coriander \\
\hline
\end{tabular}




\begin{tabular}{|c|c|c|c|}
\hline 76. & UBC 837- (TA) $)_{8}$ RT & TATATATATATATATART & No amplification \\
\hline 77. & UBC 838- (TA) $)_{8} \mathrm{RC}$ & TATATATATATATATARC & No amplification \\
\hline 78. & UBC 839- $(\mathrm{TA})_{8} \mathrm{RG}$ & TATATATATATATATARG & No amplification \\
\hline 79. & $\mathrm{UBC} 840-(\mathrm{GA})_{8} \mathrm{YT}$ & GAGAGAGAGAGAGAGAYT & Fenugreek, Coriander \\
\hline 80. & UBC 841- $(\mathrm{GA})_{8} \mathrm{YC}$ & GAGAGAGAGAGAGAGAYC & Turmeric, Ginger, Fenugreek, Coriander \\
\hline 81. & UBC 842- $(\mathrm{GA})_{8} \mathrm{YG}$ & GAGAGAGAGAGAGAGAYG & Turmeric, Ginger, Fenugreek, Coriander \\
\hline 82. & UBC 843- $(\mathrm{CT})_{8} \mathrm{RA}$ & CTCTCTCTCTCTCTCTRA & Fenugreek, Coriander \\
\hline 83. & UBC 844- $(\mathrm{CT})_{8} \mathrm{RC}$ & CTCTCTCTCTCTCTCTRC & Fenugreek, Coriander \\
\hline 84. & UBC $845-(\mathrm{CT})_{8} \mathrm{RG}$ & СТCTCTCTCTCTCTCTRG & Ginger, Fenugreek, Coriander \\
\hline 85. & UBC 846- $(\mathrm{CA})_{8} \mathrm{RT}$ & CACACACACACACACART & Fenugreek \\
\hline 86. & UBC 847- $(\mathrm{CA})_{8} \mathrm{RC}$ & CACACACACACACACARC & Fenugreek \\
\hline 87. & UBC 848- $(\mathrm{CA})_{8} \mathrm{RG}$ & CACACACACACACACARG & Fenugreek \\
\hline 88. & UBC 849- $(\mathrm{GT})_{8} \mathrm{YA}$ & GTGTGTGTGTGTGTGTYA & Fenugreek \\
\hline 89. & UBC 850- $(\mathrm{GT})_{8} \mathrm{YC}$ & GTGTGTGTGTGTGTGTYC & Turmeric, Ginger, Coriander, Fenugreek \\
\hline 90. & UBC $851-(\mathrm{GT})_{8} \mathrm{YG}$ & GTGTGTGTGTGTGTGTYG & Turmeric, Ginger, Coriander, Fenugreek \\
\hline 91. & UBC 852- (TC) $)_{8}$ RA & TCTCTCTCTCTCTCTCRA & Ginger, Fenugreek, Coriander \\
\hline 92. & UBC 853- (TC) ${ }_{8} \mathrm{RT}$ & TCTCTCTCTCTCTCTCRT & Fenugreek \\
\hline 93. & UBC 854- (TC) $)_{8} R G$ & TCTCTCTCTCTCTCTCRG & Fenugreek \\
\hline 94. & UBC 855- $(\mathrm{AC})_{8} \mathrm{YT}$ & ACACACACACACACACYT & Turmeric, Ginger, Fenugreek \\
\hline 95. & UBC 856- $(\mathrm{AC})_{8} \mathrm{YA}$ & ACACACACACACACACYA & Turmeric, Ginger, Coriander, Fenugreek \\
\hline 96. & UBC 857- $(\mathrm{AC})_{8} \mathrm{YG}$ & ACACACACACACACACYG & Turmeric, Ginger, Coriander, Fenugreek \\
\hline 97. & UBC 858- (TG) $)_{8} \mathrm{RT}$ & TGTGTGTGTGTGTGTGRT & Turmeric, Ginger, Coriander, Fenugreek \\
\hline 98. & UBC 859- (TG) $)_{8} R G$ & TGTGTGTGTGTGTGTGRG & Coriander \\
\hline 99. & UBC 860- $(\mathrm{TG})_{8} \mathrm{RA}$ & TGTGTGTGTGTGTGTGRA & Turmeric, Ginger, Coriander, Fenugreek \\
\hline 100. & UBC $861-(\mathrm{ACC})_{6}$ & ACCACCACCACCACCACC & Coriander \\
\hline 101. & UBC 862- $(\mathrm{AGC})_{6}$ & AGCAGCAGCAGCAGCAGC & Coriander \\
\hline 102. & UBC 863- $(\mathrm{AGT})_{6}$ & AGTAGTAGTAGTAGTAGT & Coriander \\
\hline 103. & UBC 864- (ATG) ${ }_{6}$ & ATGATGATGATGATGATG & Turmeric, Ginger, Coriander \\
\hline 104. & UBC 865- $(\mathrm{CCG})_{6}$ & CCGCCGCCGCCGCCGCCG & Coriander, Fenugreek \\
\hline 105. & UBC 866- $(\mathrm{CTC})_{6}$ & СТССТССТССТССТССТС & Turmeric, Ginger, Fenugreek \\
\hline 106. & UBC 867- $(\mathrm{GGC})_{6}$ & GGCGGCGGCGGCGGCGGC & Fenugreek \\
\hline 107. & UBC 868- $(\mathrm{GAA})_{6}$ & GAAGAAGAAGAAGAAGAA & Turmeric, Ginger, Coriander \\
\hline 108. & UBC 869- $(\mathrm{GTT})_{6}$ & GTTGTTGTTGTTGTTGTT & Fenugreek, Coriander \\
\hline 109. & $\mathrm{UBC} 882-\mathrm{VBV}(\mathrm{AT})_{7}$ & VBVATATATATATATAT & No amplification \\
\hline 110. & UBC 883- (TA) $)_{7} \mathrm{BVB}$ & TATATATATATATABVB & No amplification \\
\hline 111. & $\mathrm{UBC} 884-\mathrm{HBH}(\mathrm{AG})_{7}$ & HBHAGAGAGAGAGAGAG & Ginger, Fenugreek \\
\hline 112. & UBC 888- $(\mathrm{CA})_{6} \mathrm{BDB}$ & CACACACACACABDB & No amplification \\
\hline 113. & UBC $889-(\mathrm{AC})_{8} \mathrm{DBD}$ & ACACACACACACACACDBD & No amplification \\
\hline 114. & UBC 890- (CT) $)_{7} \mathrm{VHV}$ & СТСТСТСТСТСТСТVHV & No amplification \\
\hline 115. & UBC 891- HVH(TG) & HVHTGTGTGTGTGTGTG & Turmeric \\
\hline 116. & UBC 894 & TGGTAGCTCTTGATCANNNNN & Turmeric, Ginger \\
\hline 117. & UBC 896 & AGGTCGCGGCCGCNNNNNNATG & No amplification \\
\hline 118. & UBC 897 & CCGACTCGAGNNNNNNATGTGG & Turmeric, Ginger, Fenugreek \\
\hline
\end{tabular}



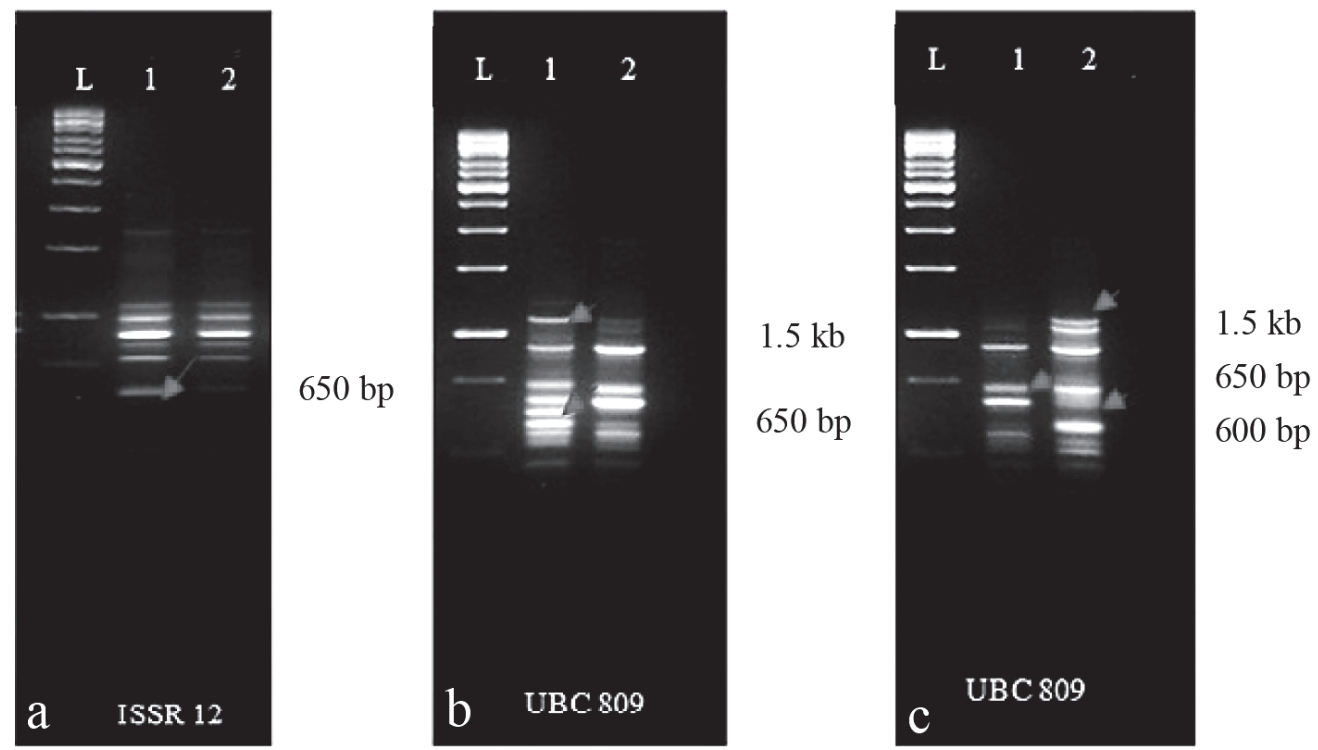

Fig. 1. a,b,c - DNA fingerprint generated for turmeric varieties using ISSR primers (ISSR 12, UBC 809). 1. a: L-1 kb ladder, 1-Suranch, 2-Roma, 1. b:L-1 kb ladder, 1-Lakadong, 2-Rasmi, 1. c: L-1kb ladder, 1-Suroma, 2-Prathibha

In the case of turmeric, primers $(\mathrm{CAC})_{3} \mathrm{GC}$ (ISSR 12), (AG) ${ }_{8} \mathrm{G}$ (UBC 809), and (GAA) 6 (UBC $868)$ produced distinct profiles. The primer ISSR 12 could effectively distinguish the candidate variety Suranch and check variety Roma by the marker ISSR $12_{650^{\circ}}$. The primer UBC 809 distinguished the candidate varieties Rasmi and Suroma from their check varieties. The polymorphic bands UBC $809_{1500,650}$ could distinguish the varieties Rasmi and Lakadong, and the markers UBC $809_{1500,650,600}$ could distinguish between the varieties Suroma and Prathibha (Fig. 1 a-c). Similarly, UBC 868 successfully identified the candidate varieties Uttara Ranjini (TCP-129) and Uttara Rupanjana (TCP-64) from the respective check varieties (TCP161 and TCP-191). The markers UBC $868_{900,750,600.450}$ distinguished the variety TCP-129 and UBC $868_{700,600,550,500,400}$ identified the variety TCP-64 from the respective check varieties (Fig. 2 a-b). Previously characterization of turmeric germplasm using ISSR primers was carried out by Syamkumar (2008). In a similar report, 19 ISSR primers were used to produce genetic fingerprints of turmeric varieties from northeast India (Das et al., 2011). Eighteen popular turmeric varieties in Telangana were analysed using ISSR primers, and some of the primers showed higher polymorphism across different genotypes (Prasanth et al., 2015). Verma et al. (2015) analysed variability among the indigenous varieties of turmeric using ISSR primers.

Fingerprinting of the ginger varieties were carried out using the ISSR primer $(\mathrm{CT}){ }_{8} \mathrm{CC}$. The primer $(\mathrm{CT})_{8} \mathrm{CC}$ distinguished the candidate varieties Suruchi, Suravi and Suprabha from the respective check varieties

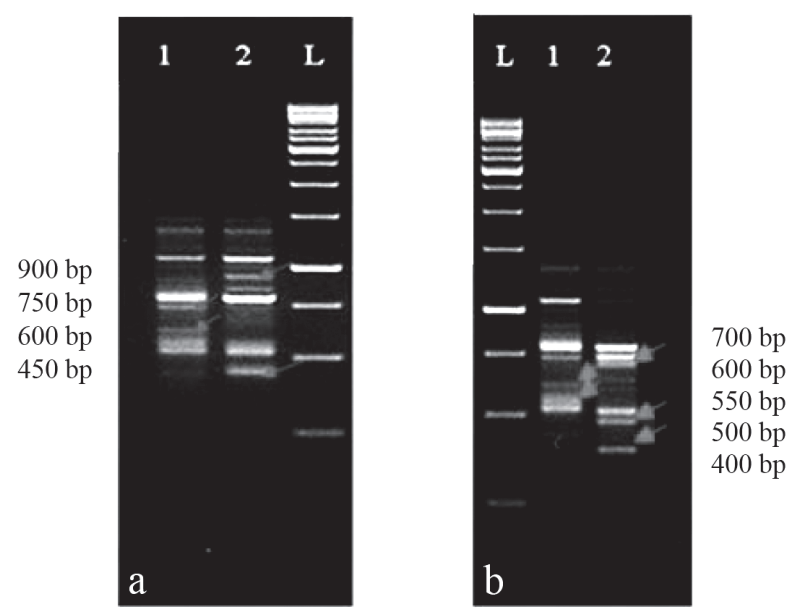

Fig. 2. a,b - DNA fingerprint generated for turmeric varieties using ISSR primers (UBC 868). 2. a: L-1kb ladder, 1-TCP-129, 2-TCP-161, 2.b: L-1kb ladder, 1-TCP-64, 2-TCP-191 

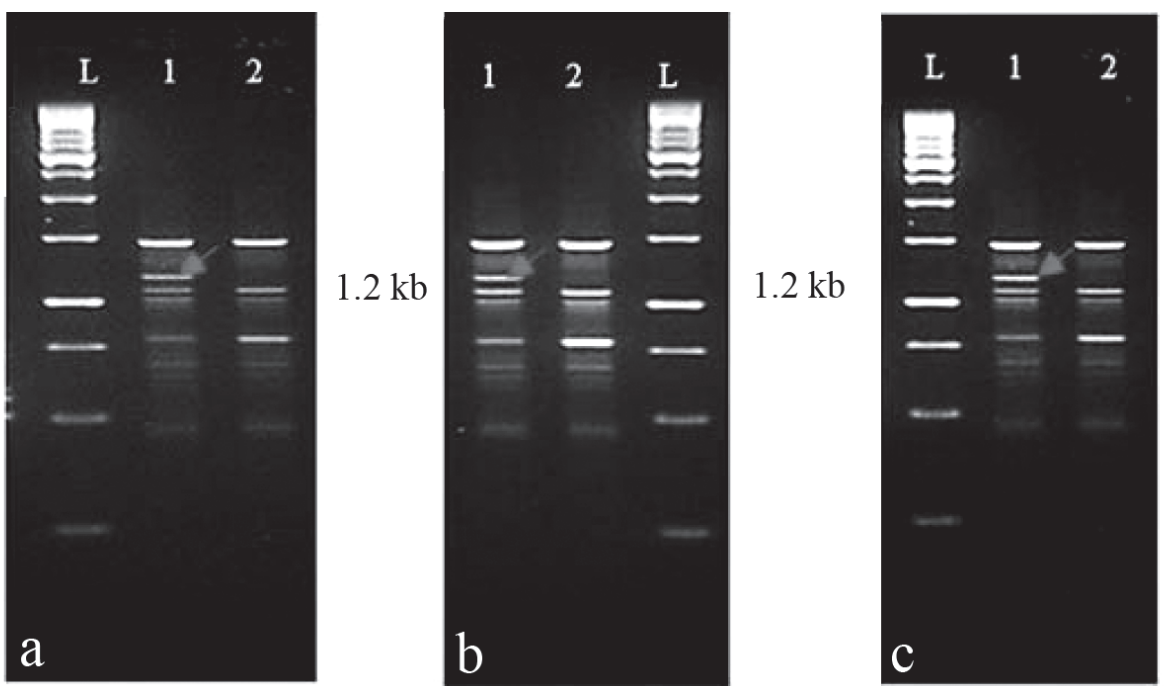

$1.2 \mathrm{~kb}$

Fig. 3. a,bc - DNA fingerprint generated for ginger varieties using ISSR primer (CT) ${ }^{8} \mathrm{CC}$ ). 3. a: L-1 kb ladder, 1-Surchi, 2 ISSR-Varada, 3. b: L-kb ladder, 1-Suravi, 2-IISR-Mahima, 3. c:L-1 kb ladder, 1-Suprabha, 2-Kundali local

IISR Varada, IISR Mahima and Kundali local respectively. The unique marker $(\mathrm{CT})_{8} \mathrm{CC}_{1200}$ was present in the candidate varieties, and the same was absent in the check varieties (Fig. 3 a-c). About 60 ginger cultivars from eastern India were analysed for their genetic diversity using ISSR primers, and it was found that ISSR primers were successful in distinguishing all the cultivars (Das et al., 2017). A report by Kizhakkayil and Sasikumar (2010) showed that ginger accessions from germplasm collection could be grouped based on analysis conducted using ISSR primers.

The primer (CAC) ${ }_{3} \mathrm{GC}$ (ISSR 12) was also used to distinguish the fenugreek varieties $\mathrm{AFg}-3, \mathrm{AFg}-4$

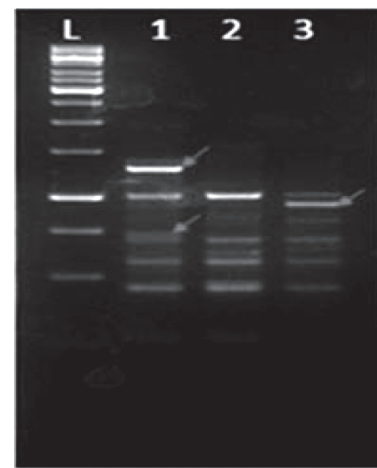

$$
\begin{aligned}
& 1.4 \mathrm{~kb} \\
& 900 \mathrm{bp} \\
& 750 \mathrm{bp}
\end{aligned}
$$

Fig. 4 DNA fingerprint generated for fenugreek varieties using ISSR primer (ISSR 12). L-1 kb ladder, 1-Afg-3, 2-Afg-4, 3-Afg-5 and AFg-5. The markers ISSR $12_{1400,900,750}$ distinguished the candidate variety $\mathrm{AFg}-5$ from the closely related check varieties AFg-3 and AFg-4 (Fig. 4). ISSR markers were used to determine the genetic diversity among fenugreek varieties, and it was found that the varieties studied were genetically diverse across different geographical locations (Mamatha et al., 2017).

Three different primers, (CAC) ${ }_{3} \mathrm{GC}$ (ISSR 12), (GA) ${ }_{8}$ YT (UBC 840) and (CTC) 6 (UBC 866) were found to generate distinct markers in varieties of coriander. The marker ISSR $12_{400}$ could identify the candidate variety $\mathrm{ACr}-2$ from its closely related varieties $\mathrm{ACr}-1$ and $\mathrm{AGCr}-1$ (Fig. 5). Likewise,

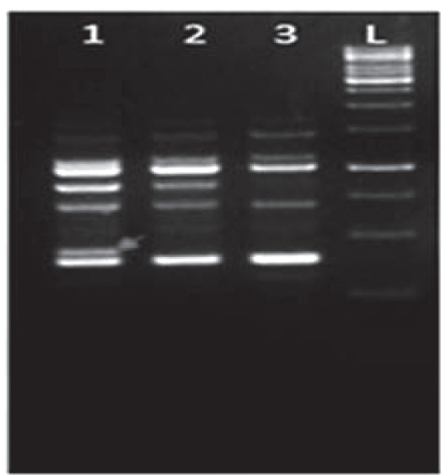

$400 \mathrm{bp}$

Fig. 5 DNA fingerprint generated for coriander varieties using ISSR primer (ISSR 12). L-1 kb ladder, 1-ACr-2, 2-ACr-1, 3-AGCr-1 

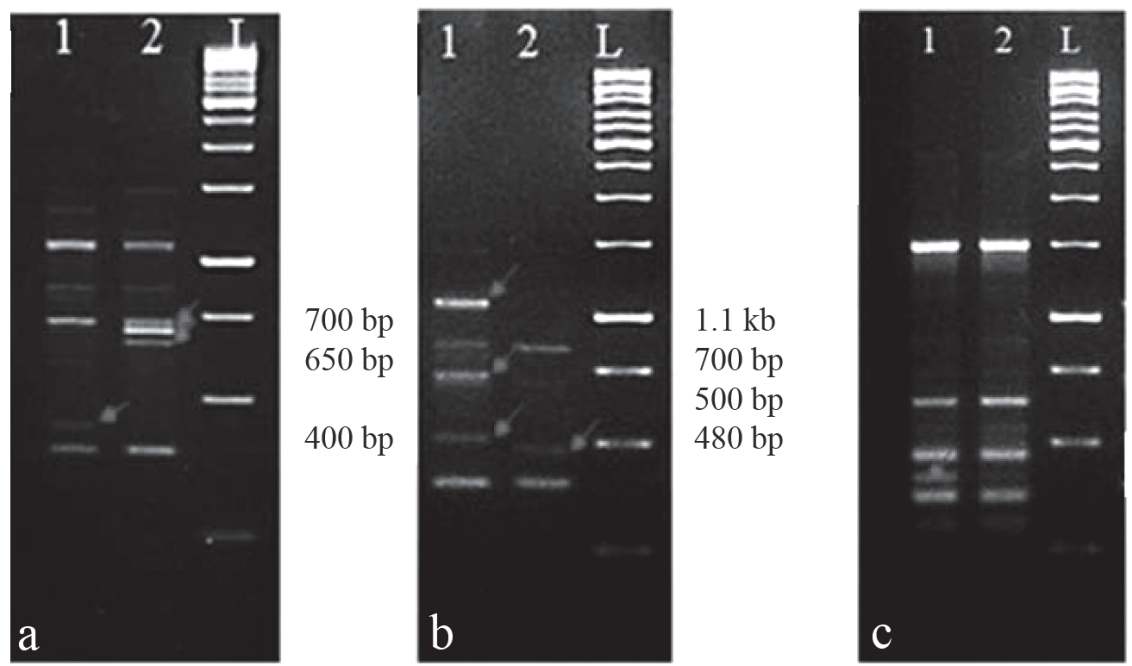

$400 \mathrm{bp}$

Fig. 6. a, b, c - DNA fingerprint generated for coriander varieties using ISSR primers (ISSR 12, UBC 840). 6. a:1-Suruchi, 2-Sindhu, L-1kb ladder, 6. b:1Susthira, 2-AD-1, L-1kb ladder, 6. c:1-Suguna, 2-Sudha, L-1kb ladder

polymorphic bands ISSR $12_{700,650,400}$ distinguished Suruchi from its check Sindhu. Marker bands ISSR $12_{1100,700,500,480}$ differentiated the candidate variety Susthira from its closely related check AD-1 and primer UBC 840 identified the candidate variety Suguna from the check variety Sudha by the presence of a unique marker UBC $840_{400}$ (Fig. 6 a-c). Fingerprinting of the candidate variety ICS-4 and the check varieties ICS-1, RCR-728 and
Hisar Anand of coriander was done using the primer combination ISSR 12 and UBC 866. The candidate variety Chhattisgarh Shri Chandra Hasini dhaniya-2 (ICS-4) and check variety Hisar Anand generated similar profiles when fingerprinted using the primer ISSR 12 and the bands ISSR 12 were common. Therefore, primer UBC 866 was used in combination with ISSR 12 for distinguishing the candidate variety ICS-4 from Hisar Anand. The band
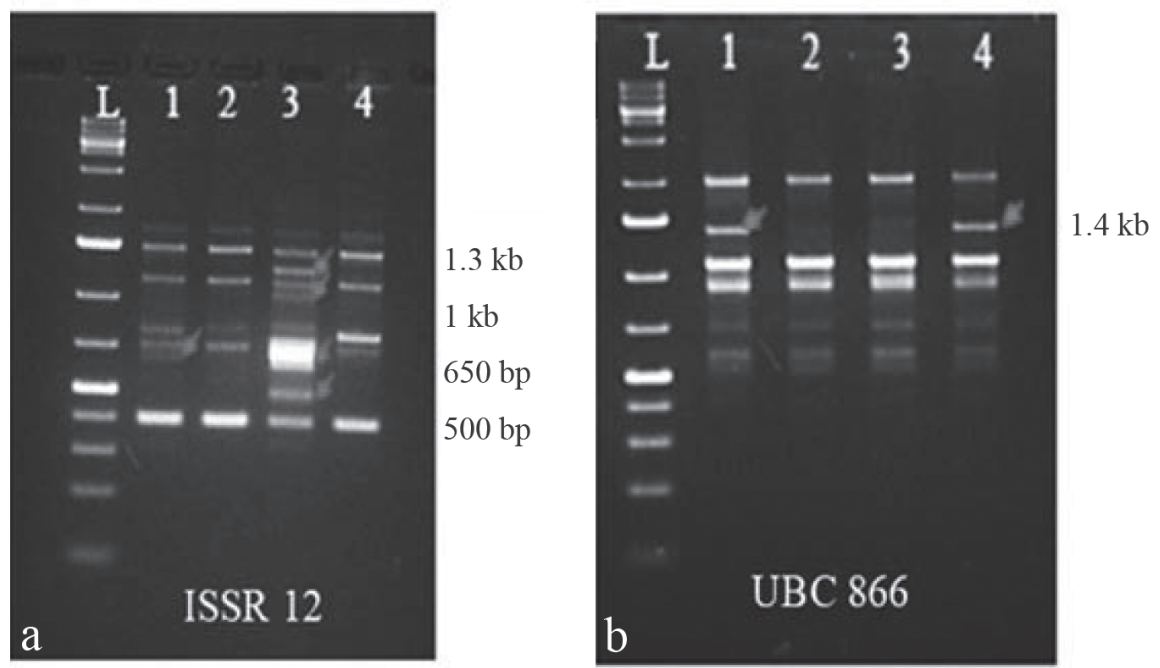

Fig. 7. a, b - DNA fingerprint generated for coriander varieties using ISSR primers (7. a-ISSR 12, 7. b-UBC 866). L-1 kb plus ladder, 1-ICS-1, 2-ICS-4, 3-RCR 728, 4-Hisar Anand 
Giridhari et al.

\begin{tabular}{|c|c|c|c|c|c|c|c|c|c|c|c|c|c|c|}
\hline 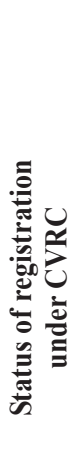 & 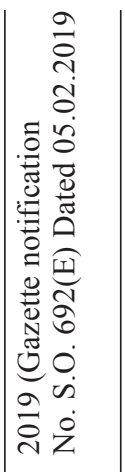 & 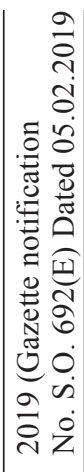 & 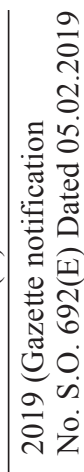 & 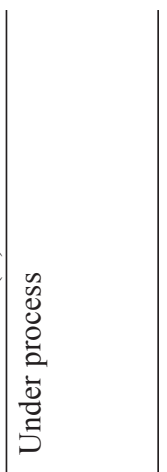 & 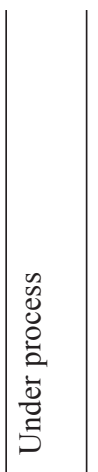 & 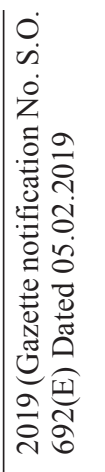 & 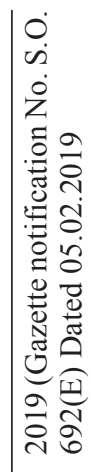 & 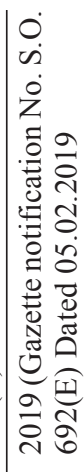 & 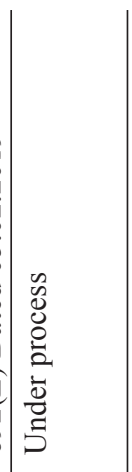 & $\begin{array}{l}0 \\
0 \\
0 \\
0 \\
0 \\
\dot{0} \\
\dot{0} \\
\overrightarrow{0}\end{array}$ & 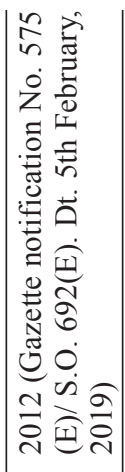 & 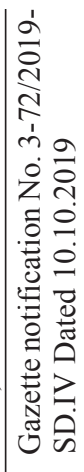 & 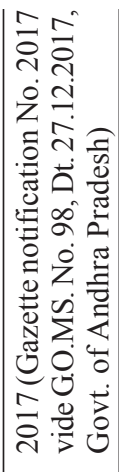 & 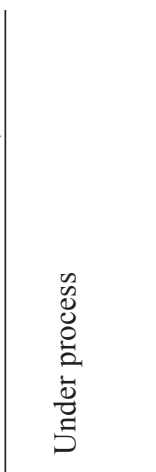 \\
\hline 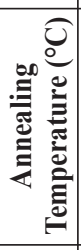 & in & ñ & "n & $\ddot{\dot{\gamma}}$ & $\ddot{\dot{\gamma}}$ & $\begin{array}{l}n \\
\infty \\
\infty \\
+\end{array}$ & $\begin{array}{l}n \\
\infty \\
\infty \\
+\end{array}$ & $\begin{array}{l}n \\
\infty \\
\infty \\
+\end{array}$ & $\begin{array}{l}n \\
6 \\
6 \\
+\end{array}$ & no & 奋 & 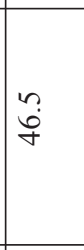 & 旾 & \begin{tabular}{l}
$n$ \\
$\ddot{0}$ \\
\multirow{+}{*}{} \\
$\dot{n}$ \\
$i n$
\end{tabular} \\
\hline 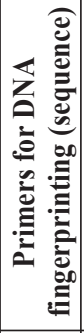 & 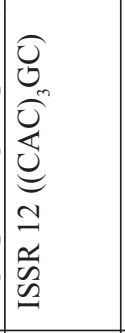 & $\begin{array}{l}\widehat{0} \\
0 \infty \\
0 \\
\vdots \\
\vdots \\
o \\
\infty \\
0 \\
0 \\
5\end{array}$ & 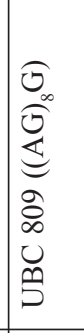 & 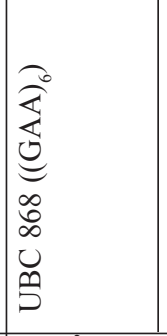 & 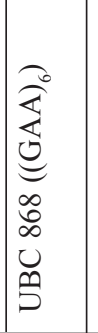 & 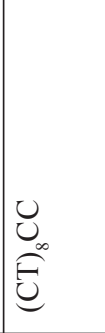 & 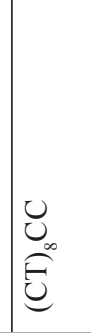 & $\begin{array}{l}\tilde{U}^{-\infty} \\
\widehat{\theta}^{-\infty}\end{array}$ & 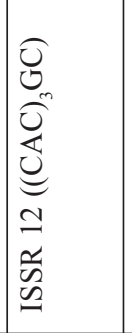 & 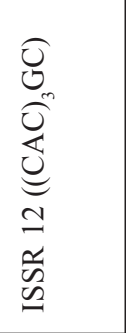 & 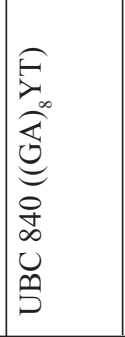 & 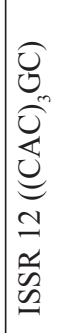 & 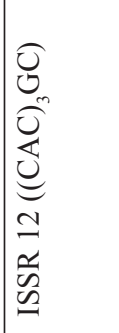 & 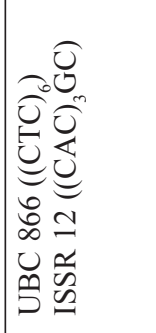 \\
\hline 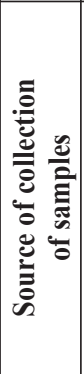 & 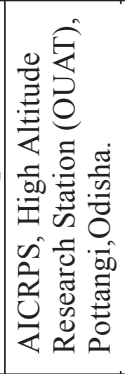 & & & 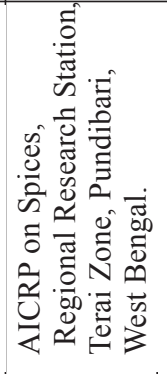 & & 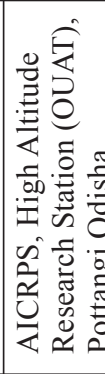 & & & 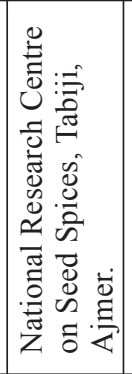 & 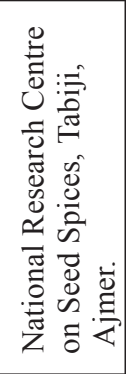 & 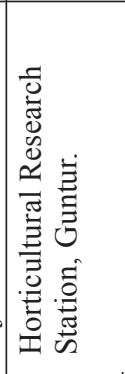 & & & 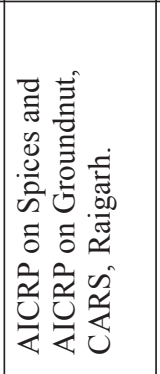 \\
\hline 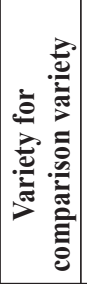 & 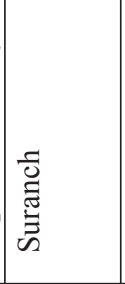 & 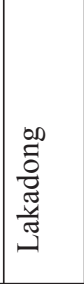 & 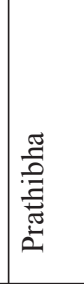 & $\begin{array}{l}\vec{\partial} \\
\overrightarrow{\tilde{U}} \\
\vec{b}\end{array}$ & $\begin{array}{l}\vec{\sigma} \\
\overrightarrow{\tilde{D}} \\
\forall\end{array}$ & 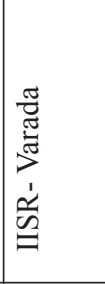 & 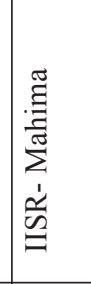 & 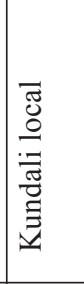 & 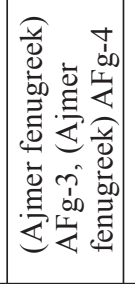 & 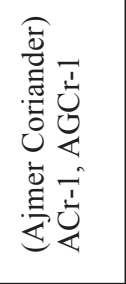 & \begin{tabular}{|l}
$\mid \frac{\pi}{7}$ \\
$\bar{z}$ \\
$\bar{n}$
\end{tabular} & $\vec{e}$ & 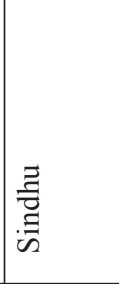 & 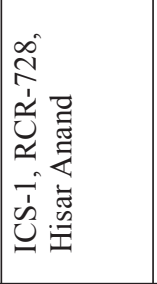 \\
\hline 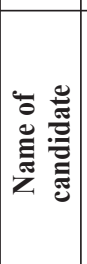 & 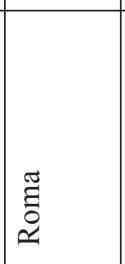 & 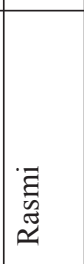 & 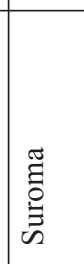 & 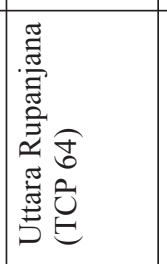 & 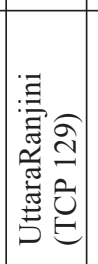 & : & 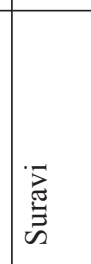 & 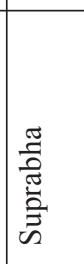 & 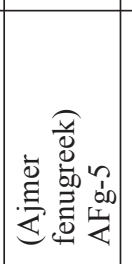 & 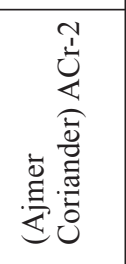 & $\begin{array}{l}\tilde{a} \\
\stackrel{\vec{E}}{50} \\
\overrightarrow{\tilde{D}}\end{array}$ & 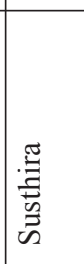 & 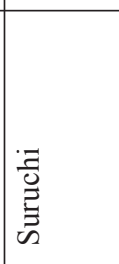 & 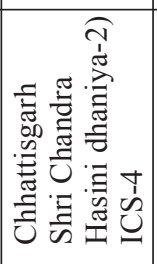 \\
\hline ?ి & 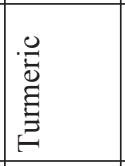 & 兽 & 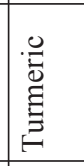 & 兽 & 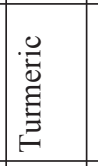 & 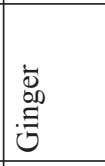 & 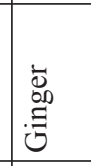 & $\begin{array}{l}\dot{\bar{\Delta}} \\
\stackrel{0}{0} \\
\dot{\Xi}\end{array}$ & 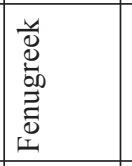 & 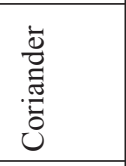 & 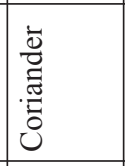 & 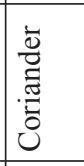 & 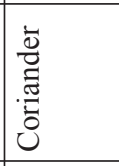 & 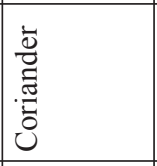 \\
\hline$\dot{\omega} \dot{z}$ & $\mid-\dot{ }$ & i & $\dot{n}$ & I & in & 6 & $\therefore$ & $\infty$ & a & $\stackrel{0}{0}$ & $\doteq$ & I & $\dot{\Omega}$ & $\dot{ \pm}$ \\
\hline
\end{tabular}


UBC $866_{1400}$ distinguished the variety ICS-4 from Hisar Anand owing to its presence in Hisar Anand and absence in ICS-4. The check varieties ICS-1, RCR-728 could be distinguished from the candidate variety using primer ISSR 12 . The markers ISSR $12_{1300,1000,500}$ were unique to the check variety RCR-728 and therefore, distinguishable. Likewise, absence of the unique bands ISSR $12_{1300,1000,500}$ and presence of the band ISSR $12{ }_{650}$, common for ICS- 1 and RCR-728 distinguished the varieties ICS-1 from RCR-728 and ICS-4 (Fig. 7 a, b). ISSR markers were used to study the genetic variability of coriander cultivars grown in Egypt (Abou El-Nasr et al., 2013).

Based on the DNA profile data generated at the DNAFF (ICAR-IISR), nine varieties of spices were notified by CVRC, while the remaining five are in the process of getting notified (Table 2).

\section{Conclusion}

Due to the availability of advanced techniques in crop manipulation and development, a large number of new varieties are being generated, and it has become increasingly difficult to differentiate these new varieties based on observable phenotypic characteristics. DNA fingerprinting is probably the only method for accurate identification of varieties in such cases, and DNA profiles of candidate vis-a-vis check variety are now mandatory for varietal registration by CVRC. Here we are suggesting an easy method for establishing distinctness for plant variety registration in spices by comparing the ISSR profiles of the candidate variety and the closely resembling check variety to identify presence or absence of unique markers. Though the method is a viable and low cost, scaling up the technique is quite challenging starting from sample collection to transportation to method of identification. Hence sincere efforts need to be taken to improve accuracy and usefulness coupled with a low cost of analysis so that the technique can be effectively integrated into the existing agricultural system for varietal identification. It is also required to make concerted effort involving scientists, breeders, statisticians, economists, computer specialists along with legal and policy experts, to chalk out the SOPs for employing plant DNA fingerprints as legal evidence, without which judicious exploitation of genetic resources for financial benefits will be difficult.

\section{References}

Abdulla, M. and Gamal, O. 2010. Investigation on molecular phylogeny of some date palm (Phoenix dactylifra L.) cultivars by protein, RAPD and ISSR markers in Saudi Arabia. Australian Journal of Crop Science 4(1): 23-28.

Abou El-Nasr, T.H.S., Ibrahim, M.M., Aboud, K.A. and El-Enany, M.A.M. 2013. Assessment of genetic variability for three coriander (Coriandrum sativum L.) cultivars grown in Egypt, using morphological characters, essential oil composition and ISSR markers. World Applied Sciences Journal 25(6): 839-849.

Alhasnawi, A.N., Amar, M.M. and Jasim, H.M. 2019. Using DNA fingerprinting to detect the genetic relationships in Acacia by inter-simple sequence repeat markers. Journal of Pure and Applied Microbiology 13(1): 281-288.

Awasthi, A.K., Nagaraja, G. and Naik, G. 2004. Genetic diversity and relationships in mulberry (genus Morus) as revealed by RAPD and ISSR marker assays. BMC Genetics 5:1. https://doi.org/10.1186/1471-2156-5-1.

Bhat, K.V. 2006. DNA Fingerprinting and Cultivar Identification. National Centre on DNA Fingerprinting, NBPGR, New Delhi.101-109 p.

Costa, R., Pereira, G., Garrido, I., Tavares-de-Sousa, M.M. and Espinosa, F. 2016. Comparison of RAPD, ISSR, and AFLP molecular markers to reveal and classify Orchard grass (Dactylis glomerata L.) germplasm variations. PLoS one 11(4): e0152972. https://doi.org/10.1371/ journal.pone.0152972.

Das, A., Gaur, M., Barik, D.P. and Subudhi, E. 2017. Genetic diversity analysis of 60 ginger germplasm core accessions using ISSR and SSR markers. Plant Biosystem 151(5): 822-832.

Das, A., Kesari, V., Satyanarayana, V.M., Parida, A. and Rangan, L. 2011. Genetic relationship of Curcuma species from Northeast India using PCR-based markers. Molecular Biotechnology 49(1): 65-76.

Doyle, J. and Doyle, J.L. 1987. Genomic plant DNA preparation from fresh tissue-CTAB method. Phytochemical Bulletin 19(11): 11-15.

Godwin, I.D., Aitken, E.A. and Smith, L.W. 1997. Application of inter simple sequence repeat (ISSR) markers to plant genetics. Electrophoresis 18(9): 1524-1528.

Gosh, P. 2013. DNA fingerprinting of released varieties and selected superior somaclones of ginger (Zingiber officinale Rosc). M.Sc Thesis, KAU, Thrissur, Kerala, India. 
Jeffreys, A.J., Wilson, V. and Thein, S.L. 1985. Individualspecific 'fingerprints' of human DNA. Nature 316(6023): 76-79.

Kizhakkayil, J. and Sasikumar, B. 2010. Genetic diversity analysis of ginger (Zingiber officinale Rosc.) germplasm based on RAPD and ISSR markers. Scientia Horticulturae 125: 73-76.

Mamatha, N.C., Tehlan, S.K., Srikanth, M., Ravikumar, T., Yashveer, S. and Mukesh, K. 2017. Assessment of genetic diversity among Fenugreek (Trigonella foenum-graecum L.) genotypes using ISSR markers. International Journal of Current Microbiology and Applied Sciences 6(6): $2565-2572$

McGregor, C., Lambert, C., Greyling, M., Louw, J.H. and Warnich, L. 2000. A comparative assessment of DNA fingerprinting techniques (RAPD, ISSR, AFLP and SSR) in tetraploid potato (Solanum tuberosum L.) germplasm. Euphytica 113: 135-144.

Nybom, H., Weising, K. and Rotter, B. 2014. DNA fingerprinting in botany: past, present, future. Investigative Genetics 5(1): 1. doi:10.1186/2041-2223-5-1.

Pasqualone, A., Lotti, C., Bruno, A., De Vita, P., Di Fonzo, N. and Blanco, A. 2000. Use of ISSR markers for cultivar identification in durum wheat. In: Durum wheat Improvement in the Mediterranean Region: New Challenges. (Eds.) Royo, C., Nachit M., Di Fonzo N. and Araus J.L. CIHEAM, Zaragoza, Spain, pp. 157-161.

Prasanth, N., Yugander, N. and Bhavani, N.L. 2015. DNA isolation and PCR amplification of turmeric varieties from Telangana State. International Journal of Current Microbiology and Applied Sciences 4(5): 485-490.
Sabir, J.S.M., Abo-Aba, S. and Bafeel, S. 2014. Characterization of ten date palm (Phoenix dactylifera $\mathrm{L}$.) cultivars from Saudi Arabia using AFLP and ISSR markers. C. R. Biologies 337: 6-18.

Shivakumar, M., Gireesh, C. and Ramesh, S.V. 2014. DNA finger printing-A tool for identification of cultivars. Popular Kheti 2(2): 160-162.

Swetha, V.P., Parvathy, V.A., Sheeja, T.E. and Sasikumar, B. 2014. Isolation and amplification of genomic DNA from barks of Cinnamomum spp. Turkish Journal of Biology 38(1): 151-155.

Syamkumar, S. 2008. Molecular biochemical and morphological characterization of selected Curcuma accessions. Ph D Thesis, Calicut University, Calicut, India.

Torre, M.P., García, M., Heinz, R. and Escandón, A.A. 2012. Analysis of genetic variability by ISSR markers in Calibrachoa caesia. Electronic Journal of Biotechnology 15(5): 1-12.

Verma, S., Singh, S., Sharma, S., Tewari, S.K., Roy, R.K., Goel, A.K. and Rana, T.S. 2015. Assessment of genetic diversity in indigenous turmeric (Curcuma longa) germplasm from India using molecular markers. Physiology and Molecular Biology of Plants 21(2): 233-242.

Wang, J.B. 2002. ISSR markers and their applications in plant genetics. Yi Chuan 24(5): 613-616.

Wu, Z., Fang, L., Wang, J and Shen, Y. 2009. Analysis of genetic diversity of Vitis by using ISSR markers. Acta Horticulturae 827: 125-130. 